\title{
The Analysis of the Topside Additional Layer of Martian Ionosphere Using MARSIS/Mars Express Data
}

\author{
Eojin Kim ${ }^{1,2 \dagger}$, Haingja Seo ${ }^{1}$, Joo Hyeon Kim ${ }^{1}$, Joo Hee Lee ${ }^{1,2}$, Yong Ha Kim², Gi-Hyuk Choi ${ }^{1}$, and \\ Eun-Sup Sim ${ }^{1}$ \\ ${ }^{1}$ Aerospace Convergence Technology Research Laboratory, Korea Aerospace Research Institute, Daejeon 305-806, Korea \\ ${ }^{2}$ Department of Astronomy and Space Science, Chungnam National University, Daejeon 305-764, Korea
}

In this study, the transient second or third layer on the topside of the Martian ionosphere were investigated with the most recently released Mars advanced radar for subsurface and ionospheric sounding/Mars Express data obtained from January 2010 to September 2011 to study the correlation between these topside additional layers and surface magnetic fields, solar zenith angle and solar activities. When examining the zones where the topside layer appeared, the occurrence rate of the topside layer was low at the areas with a strong Martian crustal magnetic field as observed by the Mars global surveyor. The occurrence rate of additional layers on the Martian topside ionosphere decreases as the solar zenith angle increases. However, these layers appeared significantly near the terminator of which solar zenith angle is $90^{\circ}$. In comparison between F10.7 which is the index of solar activities and the occurrence rate of the topside layer by date, its occurrence rate was higher in 2011 than in 2010 with less solar activities. The result of this study will contribute to better understanding of the environments in the topside of the ionosphere through the correlation between the various conditions regarding the Martian ionosphere and the transient layer.

Keywords: Mars, Martian ionosphere, Mars Express, Mars advanced radar for subsurface and ionospheric sounding, topside layer, topside sounder

\section{INTRODUCTION}

The active ionospheric sounder (AIS) of the Mars advanced radar for subsurface and ionospheric sounding (MARSIS) is the low-frequency radar onboard Mars Express orbiter. When the orbiter transmits the radio wave to the direction of Mars surface, the radio wave is reflected on the topside of Martian ionosphere and the AIS receives this radio wave signals. Originally, the satellite radar sounder was developed for observing the earth in 1960s, and it has significantly contributed to the studies on the ionosphere of the earth. Recently, the studies on the Martian ionosphere have been carried out with the radio occultation measurements, and this occultation method which the orbiter around Mars provides electron density profile along the line of sights has a disadvantage that it can only observe the range of solar zenith angle from $48^{\circ}$ to $132^{\circ}$. The MARSIS can measure the range of solar zenith angle which cannot be observed by the occultation method and provide data with high spatial resolution in comparison to the previous probes.

Generally, the ionosphere is formed of layers with horizontally concentrated electron density, and these layers reflect the radio wave of a specific frequency. When the radio wave passes through the ionosphere, it interacts with plasma and is refracted, and it is reflected at the specific frequency, and the radio wave passes through the layer without any influence of electron density at the higher frequencies. The highest frequency which is reflected at a specific altitude is called the critical frequency of the layer, and the relations between this frequency and the electron density is calculated as $f_{p}=8980 \sqrt{n_{e}} H z$ where $f_{p}$ is the (c) This is an open Access article distributed under the terms of the Creative Commons Attribution Non-Commercial License (http:// creativecommons.org/licenses/by-nc/3.0/) which premits unrestricted non-commercial use, distribution, and reproduction in any medium, provided the original work is properly cited.
Received Nov 8, 2012 Revised Nov 16, 2012 Accepted Nov 20, 2012 †Corresponding Author

E-mail: jinastro@kari.re.kr

Tel: +82-42-860-2262, Fax: +82-42-860-2269 


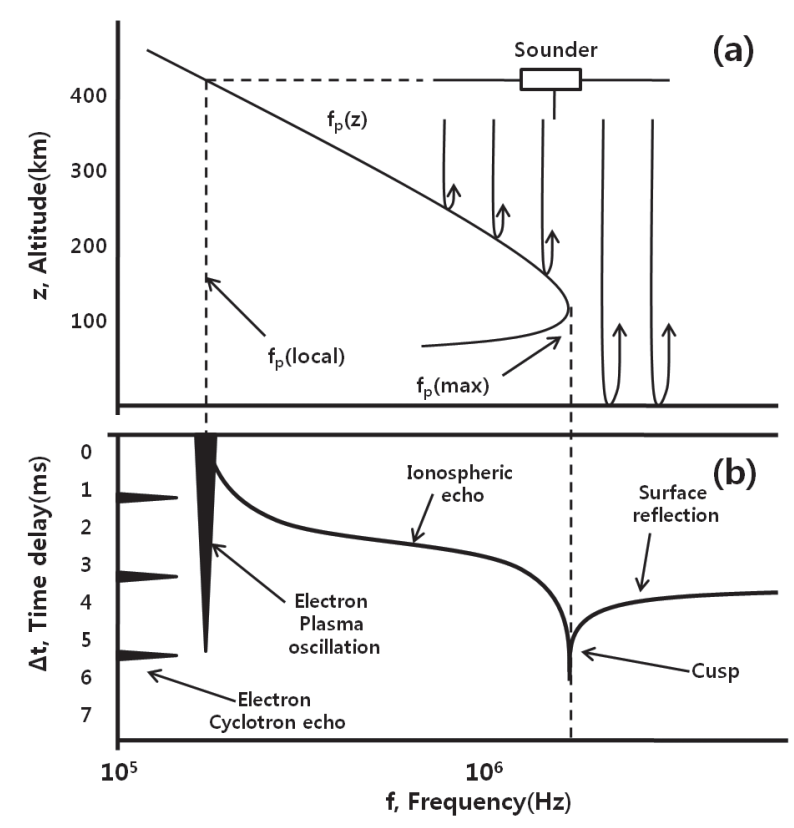

Fig. 1. Radar signal reflecting from the topside of Martian ionosphere (a) and the reflected signal features form the ionosphere and surface (b) (Gurnett et al. 2005).

critical frequency and $n_{e}$ is the electron number density (\#/ $\mathrm{cm}^{3}$ ). The MARSIS is a measuring instrument in two modes that measures the signals reflected under the surface and the signals reflected on the ionosphere. The transmitter which measures the ionosphere transmits the short pulse of frequency $\mathrm{F}$ and the MARSIS measures the strength of signal returning at each altitude of the ionosphere with time delay $\Delta t$. The transmitting frequency is increased by stages throughout the desired frequency range to measure the time delay with the function of frequency (Fig. 1a). The representation of the strength of signals provided in an image is called the ionogram, and the trace of signals corresponding to the frequency and delay time can be classified into 4 types of echo as shown in Fig. 1b. The first one is the vertical sharp echo appeared to the bottom left as spike, and it appears due to the frequency of local plasma existing around the orbiter as well as the harmonic of the vibration according to the electrostatic excitation. The second one is the signal reflected on the ionosphere, and the frequency just before the frequency which could pass through the ionosphere is the critical frequency reflected on the layer. Theoretically, when it reaches to the critical frequency, it forms cusp which the delay time becomes infinite, and it is in contact with the third signal which is reflected on the surface. The fourth one is the horizontal signal which is measured repeatedly from the cyclotron spinning of electron around the antenna due to the magnetic fields, and it is possible to calculate the strength of the crustal magnetic fields through the interval of signals. The frequency range of the AIS measurement is $100 \mathrm{kHz}-5.5$ $\mathrm{MHz}$, sweeping steps of frequency are 160, transmit-receive cycle is 91.43 micro-seconds, minimum frequency step is $10.937 \mathrm{kHz}$, and the pulse repetition frequency is $127 \mathrm{~Hz}$. The frequency sweep repetition interval is 7.38 seconds. We can measure the apparent height of signal reflected through Eq. (1) by measuring the time delay $\Delta t$ of the reflected signal returning (Gurnett et al. 2005).

$$
\Delta t(f)=\frac{2}{c} \int_{z\left(f_{p}\right)}^{z_{s c}} \frac{d z}{\sqrt{1-\left(f_{p}(z) / f\right)^{2}}}
$$

The main layer of Martian ionosphere during the daytime is $\mathrm{M} 2$ layer which mainly consists of ionized $\mathrm{CO}_{2}$, the main atmospheric species of Mars, by solar extreme ultraviolet radiation, and $\mathrm{CO}_{2}{ }^{+}$reacts with $\mathrm{O}$ atoms promptly so that $\mathrm{O}_{2}^{+}$ions is the second main ion species and it is maintained longer in its ionized status. M2 layer has the maximum electron density near the altitude of $140 \mathrm{~km}$. M1 layer exists between the altitudes of $100 \mathrm{~km}$ and $110 \mathrm{~km}$ where is 20 $\mathrm{km}$ below the M2 layer, and high energy photoelectron generated by solar X-ray collides with atmospheric atoms to create this layer. This is a process of the layer being formed by X-ray, so it changes extremely according to the level of solar activities and increase significantly during the solar flare event. A layer formed by the influx of meteoroid exists near the altitude of $85 \mathrm{~km}$. A high speed meteoroid interacts with atmospheric gas and $\mathrm{Mg}^{+}$and $\mathrm{Fe}^{+}$ions form where the meteoroid passed by, and these metallic atom ions maintain their ionic status longer than $\mathrm{O}_{2}{ }^{+}$ions. The topside above the altitude of $200 \mathrm{~km}$ is influenced by the solar radiation as well as the solar wind flux consisting of high energy particles and interplanetary magnetic fields. For the environment of magnetic fields at the altitude above topside, Martian crustal magnetic fields are different according to the areas and the magnetic fields of the solar wind incoming from the outside vary according to the location and time, so a layer occurred in the topside is also influenced by these changes (Withers 2011).

The MARSIS provides the data for vertical distribution of electron density on the topside of the Martian ionosphere, and due to the characteristics of radar, it is only possible to measure the topside of M2 layer which reflects the maximum critical frequency. The transient second or third layer observed on top of M2 which is the main layer of the Martian ionosphere. In this study, the comparative analysis between the conditions expected to influence the 


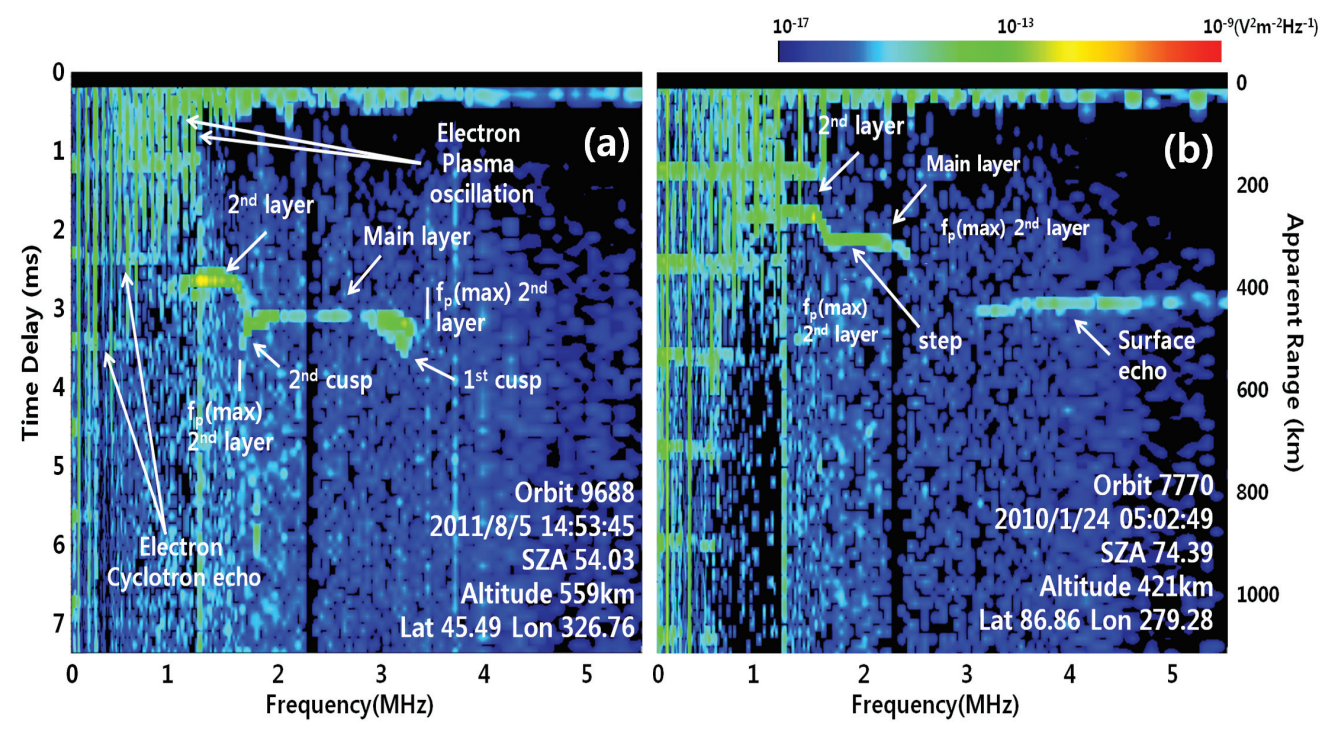

Fig. 2. Two colored ionograms including second layer observed at August 5, 2011 (a) and January 24, 2010 (b).

occurrence of this topside layer and the occurrence rate of the topside layer was carried out. Gurnett et al. (2008) suggested that the occurrence of this topside layer would be associated with the local crustal magnetic field existing on the surface of Mars, and the correlation between the occurrence rate of the topside layer and the crustal magnetic fields was analyzed and examined through the analysis of the occurrence rate of the topside layer by location in this study. And also, if we assume the creation of Chapman layer due to photoionization, it is expected that the occurrence of the topside layer will decrease as the solar zenith angle increases. And, the correlation between the occurrence rate of the topside layer and F10.7 which is the solar activity index was analyzed. Section 2 deals with the observation method of the AIS and the characteristics of data, Section 3 contains the results of topside layer data analysis, and Section 4 discusses the results of data analysis in Section 3.

\section{DATA AND METHODS}

The Mars Express orbiter of European Space Agency which was launched on June 2003 entered into an eccentric orbit around Mars on December 2003, and it is being operated until now. For the orbital elements of Mars Express, periapsis altitude is $275 \mathrm{~km}$, apoapsis altitude is $10,100 \mathrm{~km}$, orbital inclination is $86^{\circ}$, and the cycle is 6.75 hours. The MARSIS had a problem in opening its antenna in the initial stage of the Mars Express operation, so its operation became available from July 2005. MARSIS AIS measures the ionosphere for approximately 40 minutes while Mars Express approaches from the altitude of 1,200 $\mathrm{km}$ to the periapsis and recedes into $1,200 \mathrm{~km}$ altitude again. The ionosphere radar sounder data is provided in the ionogram form which represents the strength of frequency and signals by delay time in images. The data used in this paper was downloaded from http://geo.pds.nasa.gov/ missions/mars_express/marsis.htm.

Two pictures in Fig. 2 show the ionograms where the second layer appears. Fig. 2a is the ionogram observed at 14:53:45 UT on August 5, 2011, and the second layer and second cusp appear clearly. The vertical signals on the top left in the frequency less than $1.3 \mathrm{MHz}$ are the harmonics of the local electron plasma frequency, and the vibration on a low frequency where cannot be observed is shown in the harmonic form. The signal reflected on the ionospheric main layer between $1.7 \mathrm{MHz}$ and $3.5 \mathrm{MHz}$ appear within the 3-4 ms of time delay range. The topside layer is observed in the 1-2 ms of time delay range, and we can see that it has formed approximately $40 \mathrm{~km}$ higher than the main layer from the 1-1.6 MHz frequency range. The horizontal signal appeared on the left in $1 \mathrm{~ms}$ time delay interval is created by periodic signals according to the electron cyclotron around the probe due to the crustal magnetic fields of Mars. Maximum plasma frequency $\mathrm{f}_{\mathrm{p}}$ ( $\max$ ) can be decided in the frequency which forms the cusp shape where the time delay of the signal is infinite theologically, and in case of Fig. $2 a, f_{p}$ is $3.5 \mathrm{MHz}$.

Fig. $2 \mathrm{~b}$ is the ionogram observed at 05:02:49 UT on January 24,2010 , and the signal reflected on the ionospheric 
main layer is observed at $2 \mathrm{~ms}$ time delay between $1.6 \mathrm{MHz}$ and 2.8 MHz. The topside layer is observed in the range of 1-2 ms time delay, which has formed in the 1-1.6 $\mathrm{MHz}$ frequency range approximately $50 \mathrm{~km}$ higher from the main layer, and unlike Fig. 2a, the second layer in step form, not clear cusp, is connected with the main layer. As shown in the altitude of probe indicated in the figure, a strong signal reflected on the surface of Mars appears over $3 \mathrm{MHz}$ in consideration of the time delay of the signal. In case of Fig. $2 \mathrm{~b}, \mathrm{f}_{\mathrm{p}}$ is $2.5 \mathrm{MHz}$. The apparent height calculated by the Eq. (1) is displayed at the vertical axis on the right side of the ionogram in Fig. 2b.

\section{RESULTS}

The occurrence of transient additional layers on the topside of Martian ionosphere was investigated under various conditions by the analysis of the AIS data from the MARSIS. In order to exclude the cases with unclear signals first, only the cases of observation at the altitude of the probe below $1,000 \mathrm{~km}$ were considered. The topside layer was discovered from approximately 7,000 out of over 90,000 ionograms observed between $0^{\circ}$ and $120^{\circ}$ of the solar zenith angle. At this time, we only selected the cases that the altitude of the second layer was at least $30 \mathrm{~km}$ higher than M2 layer and steps or cusps appeared clearly. And also, in order to secure the accuracy of data, we excluded any case with no connected horizontal signal trace for the hook form.

At first, the occurrence rate of the topside layer according to each area bin on the surface of Mars was examined. We can see the relation between the crustal magnetic fields existing regionally on Mars and the occurrence of the topside layer. Fig. 3a shows the number of observations within each $2^{\circ} \times 2^{\circ}$ binning of latitude and longitude on Mars and Fig. 3b shows the occurrence rate of the topside layer by the number of observations in color. Gurnett et al. (2008) introduced that Shinagawa et al. (1991) suggested that the second peak of the topside occurred additionally on the topside of Martian ionosphere could be formed in case the magnetic field existed in the ionosphere. After the Mars global surveyor (MGS) mission provides magnetometer data, the researchers map the crustal magnetic fields existing on the surface of Mars. Fig. 4 is the Martian crustal magnetic field map which was introduced by Connerney et al. (2005), and this map show the level of changes in magnetic fields in the radial direction within the bin of $1^{\circ} \times 1^{\circ}$ (latitude/longitude) at the altitude of 400 $\mathrm{km}$ in Mars which was observed by MGS. Strong magnetic fields are distributed mainly in the southern hemisphere
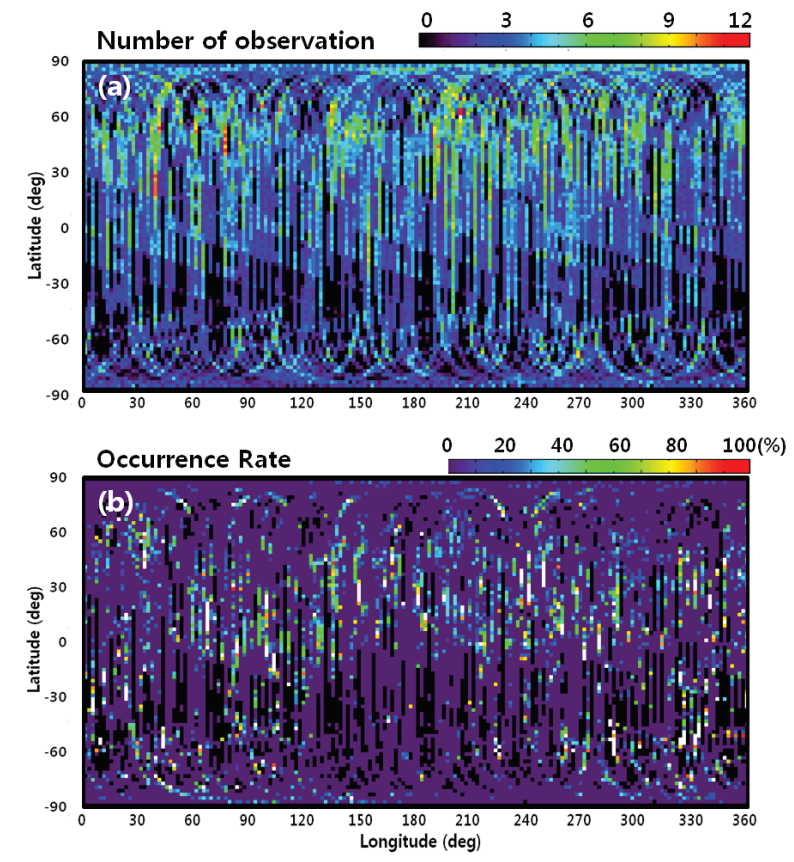

Fig. 3. (a) Number of observation, (b) occurrence rate of topside layer within $2^{\circ} \times 2^{\circ}$.

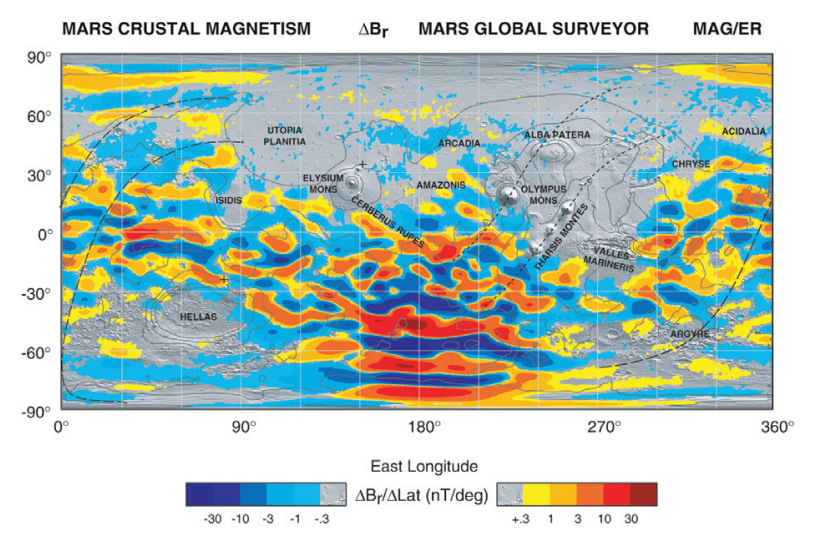

Fig. 4. Map of the magnetic field of Mars observed by the Mars global surveyor satellite at a nominal 400-km altitude (Connerney et al. 2005).

rather than the northern hemisphere, and especially the area in $90-270^{\circ} \mathrm{E}$ region of the longitude of the southern hemisphere is the area with the strongest magnetic field as \pm 3 -30 nT/deg. When we look at Fig. 3b which indicates the occurrence rate of the topside layer on the map, we can see that the occurrence rate is relatively higher at the northern hemisphere than the southern hemisphere. It is true that the data with limited observation altitude and solar zenith angle was used, and there was relatively more observation data on the northern hemisphere than the southern hemisphere due to the orbit observed by the probe from 


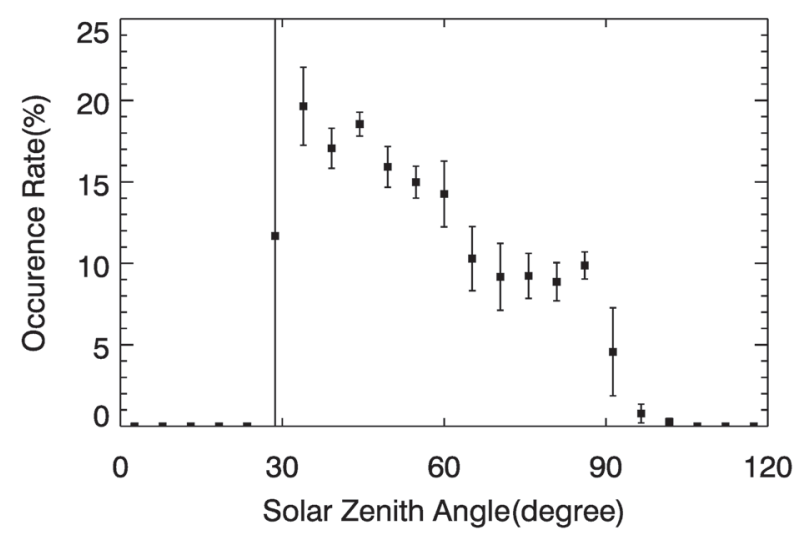

Fig. 5. Occurrence rate of topside layer with error bar as a function of solar zenith angle.

January 2010 to September 2011. However, there is no doubt that the topside layer occurred clearly less in the domain of the southern hemisphere $90-270^{\circ} \mathrm{E}$ with a strong magnetic field in consideration that the observation data of the whole southern hemisphere was compared. Kopf et al. (2008) explained that the topside layer had no association with the magnetic fields but did not provide the detailed results. In this study, the fact that the occurrence rate of the topside layer was lower in the area with a strong magnetic field than other areas was noticed through the comparison between the where the topside layer occurs and the magnetic field map even though it was not a quantitative comparison.

Next, Fig. 5 shows the occurrence rate of the topside layer by the number of the relevant observations and the error range between solar zenith angle $0^{\circ}$ and $120^{\circ}$ in a $10^{\circ}$ interval. There was no case of observation at the solar zenith angle below $21^{\circ}$ and no case of the topside layer observed between $20^{\circ}$ and $25^{\circ}$ below $1,000 \mathrm{~km}$ during the measurement period of the data used. Therefore, it is expressed that there is no occurrence rate of the topside layer under $25^{\circ}$ of the solar zenith angle. And also, the error range is very high between $25^{\circ}$ and $30^{\circ}$, which is considered unreliable. We can see at the solar zenith angle over $30^{\circ}$ that the occurrence rate decreased from $20 \%$ as the solar zenith angle gradually increased. The result of Kopf et al. (2008) at the solar zenith angle below $30^{\circ}$ cannot be compared due to the characteristics of data used in this paper, and the number of data used in Kopf et al. (2008) was 4000, which is significantly less than the number of data used in this paper, so there is a difference in the absolute value of the occurrence rate, but it shows the same result that the occurrence rate of the topside layer decreased as the solar zenith angle increased. It is significant that the occurrence rate is higher near $80^{\circ}$ of the solar zenith angle than 60 -

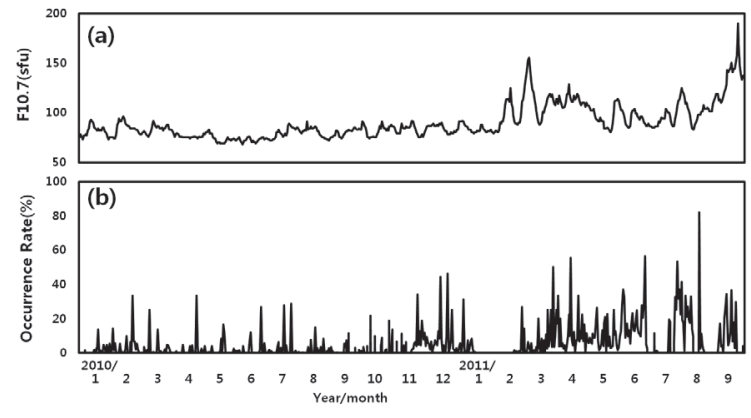

Fig. 6. Daily variation of the solar activity indices (F10.7) (a) and the occurrence rate of topside layer (b) during the period of January 2010 and September 2011.

$70^{\circ}$ of the solar zenith angle. Kopf et al. (2008) explains this that there is a high possibility of the fluctuation of plasma generated in the high ionosphere due to the instability of Kelvin-Helmholthz being occurred near the terminator where the boundary of night and day rather than the subsolar point and its occurrence rate will be high when the solar zenith angle is low. The result of this paper is similar to the result of Kopf et al. (2008), but the occurrence rate of the topside layer was relatively high near the terminator. It is necessary to consider that the number of data used in Kopf et al. (2008) is 4,000 which is significantly lesser in comparison to the number of data used in this study.

Next, the relation between the occurrence rate of the topside layer and the solar activities was examined. Fig. 6a shows F10.7 index which was daily solar activity index from January 1, 2010 to September 30, 2011, and Fig. 6b shows the number of points and the occurrence rate of the topside layer per day. In case of 2010, the F10.7 index was 100 solar flux units (sfu) which corresponded to the period of minimum solar activity, and in case of 2011, the F10.7 index was over $100 \mathrm{sfu}$ for most periods which corresponded to the period of relatively strong solar activities. We can also see that more days in 2011 have a high occurrence rate of the topside layer than 2010. However, many of data used in this study were observed in 2010 and daily change of F10.7 index in 2011 was high so it is difficult to compare between the occurrence rate of the topside layer with the F10.7 index directly. In order to supplement this, it is necessary to compare the data for a longer period of time, but the period of maximum solar activities already passed and the period between 2005 when the MARSIS operation began and 2010 is the period of solar activities being decreased, so in order to compare data in the period of strong solar activities would be possible after the release of data after September 2011. 


\section{DISCUSSION}

In this paper, the comparative analysis between the occurrence rate of the topside layer additionally appeared on the topside of the main layer of Martian ionosphere and crustal magnetic field on the surface, solar zenith angle and solar activities was carried out with the data measured from the MARSIS AIS radar onboard Mars Express.

There are additional error factors inevitably for selecting the ionogram which shows the topside layer during the analysis process. As shown in Fig. 2, the ionograms provided by the MARSIS AIS show the vertical harmonic signals appeared regularly due to the electrons near the orbiter or periodic signals appeared horizontally caused by the cyclotron motion of electrons near the antenna due to the magnetic fields so that it is difficult to distinguish actual ionospheric signals in a low frequency range. This is the characteristics of the topside sounder, and due to the characteristics of observation orbit of the Mars Express, in case of observing in a low altitude with enough solar zenith angle, these effects become larger due to the plasma existing around the probe, so it is more difficult to distinguish the signals than the case of observing in a properly high altitude, and especially the effect is significant in a low frequency domain less than 1.5 MHz. Usually oblique signal often mixes with ordinary ionospheric signals in the vertical direction in area with a strong crustal magnetic field. Due to these reasons, the cases of being unable to distinguish the signals due to plasmas near the satellite and the cases of being unable to distinguish the signals with oblique signals could act as the additional error factors for the analysis of the topside layer.

It is very interesting that the occurrence rate of the topside layers is lower in the areas with a strong magnetic field than the areas with a relatively weak magnetic field. It can be interpreted that it is possible to consider the plasmas being swept by the solar wind through the interaction between the surface magnetic fields of Mars and the magnetic fields of the solar wind rather than the layer being formed by the solar wind particles coming from the outside along the movement of plasma in the lower layer due magnetic fields in the radial direction or the magnetic fields. This sweeping of Martian ionosphere plasma by the solar wind was also verified by Yi et al. (1999) in the simulation that considered mass loading of plasma originated from Martian magnetosphere on the solar wind. This means that the comparative analysis of Mars topside data provided by MARSIS AIS and space environment plasma data of Mars such as Analyzer of Space Plasmas and Energetic Atoms-3 onboard Mars Express could contribute to the studies regarding the interaction between plasma near Mars and the solar wind. And, it can be also connected to the studies that confirm the relation between the strength of magnetic fields and the occurrence rate of the topside layer quantitatively through the calculation of magnetic field model of Mars in future. If we additionally analyze the data of period which is not used in this paper, it would be possible to obtain more accurate result from additional data for the southern hemisphere not covered in this study due to the restriction on the solar zenith angle or the observation altitude. And also, if data for below $20^{\circ}$ of the solar zenith angle is added, it would be also possible to obtain the better result for the relation between the solar zenith angle and the occurrence rate of the topside layer as well as the relation with solar activity index. The Mars Express will be operated until 2014 and solar maximum will be in 2013 , so it is expected to have interesting results regarding the Martian ionosphere based on the data obtained under the various solar activity conditions which will be provided in future.

\section{REFERENCES}

Connerney JEP, Acuna MH, Ness NF, Kletetschka G, Mitchell DL, et al., Tectonic implications of Mars crustal magnetism, PNAS, 102, 14970-14975 (2005). http:// dx.doi.org/10.1073/pnas.0507469102

Gurnett DA, Huff RL, Morgan DD, Persoon AM, Averkamp TF, et al., An overview of radar sounding of the martian ionosphere from the Mars Express spacecraft, AdSpR, 41, 1335-1346 (2008). http://dx.doi.org/10.1016/ j.asr.2007.01.062

Gurnett DA, Kirchner DL, Huff RL, Morgan DD, Persoon AM, et al., Radar soundings of the ionosphere of Mars, Sci, 310, 1929-1933 (2005). http://dx.doi.org/10.1126/ science. 1121868

Kopf AJ, Gurnett DA, Morgan DD, Kirchner DL, Transient layers in the topside ionosphere of Mars, GeoRL, 35, L17102 (2008). http://dx.doi.org/10.1029/2008GL034948

Shinagawa H, Kim J, Nagy AF, Cravens TE, A comprehensive magnetohydrodynamic model of the Venus ionosphere, JGR, 96, 11083-11095 (1991). http://dx.doi. org/10.1029/90JA02505

Withers, P, Attenuation of radio signals by the ionosphere of Mars: theoretical development and application to MARSIS observations, RaSc, 46, RS2004 (2011). http:// dx.doi.org/10.1029/2010RS004450

Yi Y, Kim E, Kim YH, Kim J, Variability of bow shock location at Mars, JASS, 16, 139-148 (1999). 